\title{
碱性磷酸酶荧光探针的研究进展
}

\author{
张继东*, $a$ 刘鸿泽 ${ }^{a}$ 孟 丽 $b$ \\ ( ${ }^{a}$ 安康学院化学化工学院 安康 725000)

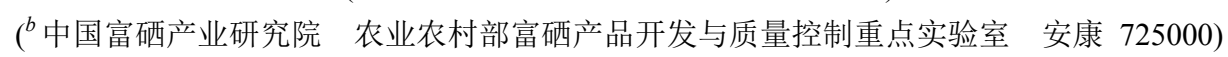

\begin{abstract}
摘要 碱性磷酸酶(ALP) 是一种广泛存在于各种哺乳动物组织中的重要酶, 作为生物标记物和诊断指标为分子生物学 的研究应用及人类疾病治疗提供了重要信息. 由于其在人类身体健康方面的信息可靠性，近年来在荧光检测技术的研 究日趋激烈, 已发展成为一类重要的检测方法. 根据不同的发光特点从(1)基于激发态分子内质子转移(ESIPT)和分子 内电荷转移型(ICT)的传感体系、(2)基于聚集诱导发光的传感体系(AIE)、(3)基于降低给电子能力(D d-E)或扰乱共轭 $\pi$ 电子的传感体系( $\mathrm{D} \pi-\mathrm{C}$ )和(4)基于苂光内滤效应(IFE)的传感体系几个方面综述了近年来 ALP 的荧光检测、活性评价及 生物成像方面的研究进展, 并对其发展趋势做了展望.
\end{abstract}

关键词＼cjkstart碱性磷酸酶; 苂光检测; 生物成像; 聚集诱导发光

\section{Research Progress in the Fluorescent Probes for Alkaline Phosphatase}

\author{
Zhang, Jidong ${ }^{*, a} \quad$ Liu, Hongze ${ }^{a} \quad$ Meng, Li $^{b}$ \\ ( ${ }^{a}$ School of Chemistry \& Chemical Engineering, Ankang Univerisity, Ankang 725000) \\ ( ${ }^{b}$ Key Laboratory of Se-Enriched Products Development and Quality Control of Ministry of Agriculture, Se-Enriched Products \\ Research Institute of China, Ankang 725000)
}

\begin{abstract}
Alkaline phosphatase (ALP) is an important enzyme for various mammalian tissues. As a biomarker and diagnostic indicator, ALP provides important information for the applied research of molecular biology and the treatment of human diseases. Due to its reliability of information in human health, the research on fluorescence detection as important detection method has become intense in recent years. The fluorescent chemosensors are categorized by different luminous feature involving excitedstate intramolecular proton transfer (ESIPT) and intramolecular charge transfer (ICT) chemosensors, aggregation-induced luminescent molecules (AIE), diminishes the electron donating ability (D d-E) and disruption of the $\pi$ conjugated systems (D $\pi$-C), inner filter effect (IFE) chemosensors, and other sensing systems. In the end, the development tendency of the sensing ensembles for ALP is prospected.

Keywords alkaline phosphatase; fluorescence detection; biological imaging; aggregation-induced emission
\end{abstract}

碱性磷酸酶(ALP)是一种哺乳动物组织中的重要酶, 具有催化磷酸酯的水解和磷酸化功能 ${ }^{[1,2]}$. 作为医疗诊 断的关键生物标记物, ALP 主要存在于肝脏、肾脏、肠 道、骨骼和胎盘中, 健康成人血清中 ALP 浓度大小范围 为 46 190 mU/mL ${ }^{[2 \sim 4]}$. 已经证实血清中 ALP 水平的异 常与各种病理过程密切相关, 例如心力衰竭、肾功能恶
化、肝功能障碍、胆汁淤积、血管钙化、骨病和乳腺癌 等疾病. 尽管人们对 ALP 进行了密切地研究, 但关于 ALP 的复杂生理功能尚未完全了解. 因此, 开发能够用 于人类血清或血液中快速、简便、灵敏检测 ALP 活性 的方法是当前研究的热点 ${ }^{[5]}$.

最近, 大量用于 ALP 活性及浓度的测定分析方法

\footnotetext{
* Corresponding author. E-mail: akuzjd@aku.edu.cn

Received March 4, 2019; revised May 31, 2019; published online June 19, 2019.

Project supported by the Key Projects of Shaanxi Provincial Science \& Technology Department (No. 2018PT-31), the Major Scientific Research Projects of the Leading Industry of Ankang City (No. 2016AKZDCY002), the Doctor's Initial Funding of Ankang University (No. 2018AYQDZR06), the Key Laboratory of Se-Enriched Products Development and Quality Control, Ministry of Agriculture (No. Se-2018B02) and the Shaanxi Provincial Innovation Experiment Program for University Students (No. 201839032).

陕西省科技厅重点(No. 2018PT-31)、安康市主导产业重大科研攻关(No. 2016AKZDCY002)、安康学院博士启动基金(No. 2018AYQDZR06)、农业部 富硒产品开发国家地方联合工程实验室开放课题(No. Se-2018B02)和陕西省大学生创新创业训练计划(No. 201839032)资助项目.
} 
被报道, 包括比色法 ${ }^{[6]}$ 、表面增强拉曼散射法 ${ }^{[7]}$ 、电化学 分析法 ${ }^{[8]}$ 和色谱分析法 ${ }^{[9]}$ 等. 在这些检测方法中, 荧光 检测技术因其固有的优势, 如高灵敏度、实时检测和原 位成像的特点而被广泛关注 ${ }^{[10,11]}$. 另外, 值得引起科学 家注意的是荧光检测方法可用于生物系统中活性物质 的检测 ${ }^{[12,13]}$. 目前, ALP 及其类似物的检测在生物医学 方面的研究逐渐成为热点, 尤其是内源性 ALP 的检测、 识别和近红外苂光成像检测等方面的研究, 为疾病的诊 断和治疗提供有力依据. 本文综述了近年来国内外对 ALP 荧光探针的研究现状, 并对未来研究发展趋势进行 展望.

\section{1 基于 ESIPT 和 ICT 的传感体系}

激发态分子内质子转移(Excited State Intramolecular Proton Transfer, ESIPT) 是激发态分子内部邻近的质子给 体(如羟基)转移到受体(如羰基), 导致互变异构体的苂 光发射存在较大的斯托克斯位移. ESIPT 是一种重要的 荧光发光现象, 化合物自身能够通过分子内的氢键诱导 荧光的转变, 其过程能够以较快的速度发生 ${ }^{[14]}$. 在水溶 液中, ESIPT 苂光分子具有低背景荧光信号的优势. 与 其它一些荧光发光过程相比较, 较大的斯托克斯位移的 存在, 使得其分子能够减少激发光的干扰. 基于 ESIPT 的 ALP 苂光检测体系, 在水溶液和生命体系中都可以 运行. 分子内电荷转移型(Intermolecular Charge Transfer, ICT) 是与 ESIPT 同等重要的苂光发光机制, 调节 ICT 过程, 导致紫外吸收和荧光发射光谱的比率型信号
出现 ${ }^{[15]}$. 比率型荧光探针采用两种荧光发射作为荧光 输出信号. 由于其结果不受自身荧光发射的干扰，因此 可以方便通过发射强度得到待测物质浓度. 近年来, ICT 发光机制被用于 ALP 荧光探针的设计中，由此实现 了 ALP 的比率型荧光检测.

2015 年, 吴水珠等 ${ }^{[16]}$ 利用 ESIPT 发光机制, 构建了 一种能够检测 ALP 的苂光探针 1 (Eq. 1). 该探针是通过 将磷酸盐官能团偶联到黄酮结构的苂光团而得到. 其磷 酸基团既可以是 ESIPT 的阻断剂, 又是 ALP 的响应基 团. 由于羟基被磷酸基团所屏蔽，所以结构本身没有 ESIPT 过程发生. 当 ALP 存在的情况下, ALP 䢃开了磷 酸基团，羟基官能团的产生导致基于 ESIPT 的苂光发射 的形成. 该探针对 ALP 表现出高的灵敏度, 其检测限为 $0.032 \mathrm{U} / \mathrm{L}$. 该探针能够应用于血清中 ALP 的检测. 通过 细胞试验研究发现, 探针能够被用于内源性 ALP 的苂 光成像. 因此, 该探针在生物诊断和病例分析中具有潜 在应用价值.

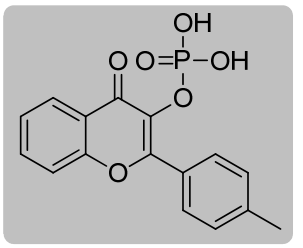

1

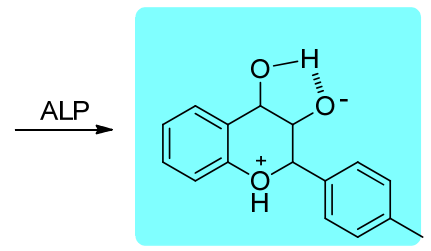

唐本忠等 ${ }^{[17]}$ 设计合成了一种基于 ESIPT 和 AIE 发 光过程的比率型苂光探针 2 (Scheme 1), 用于活细胞中
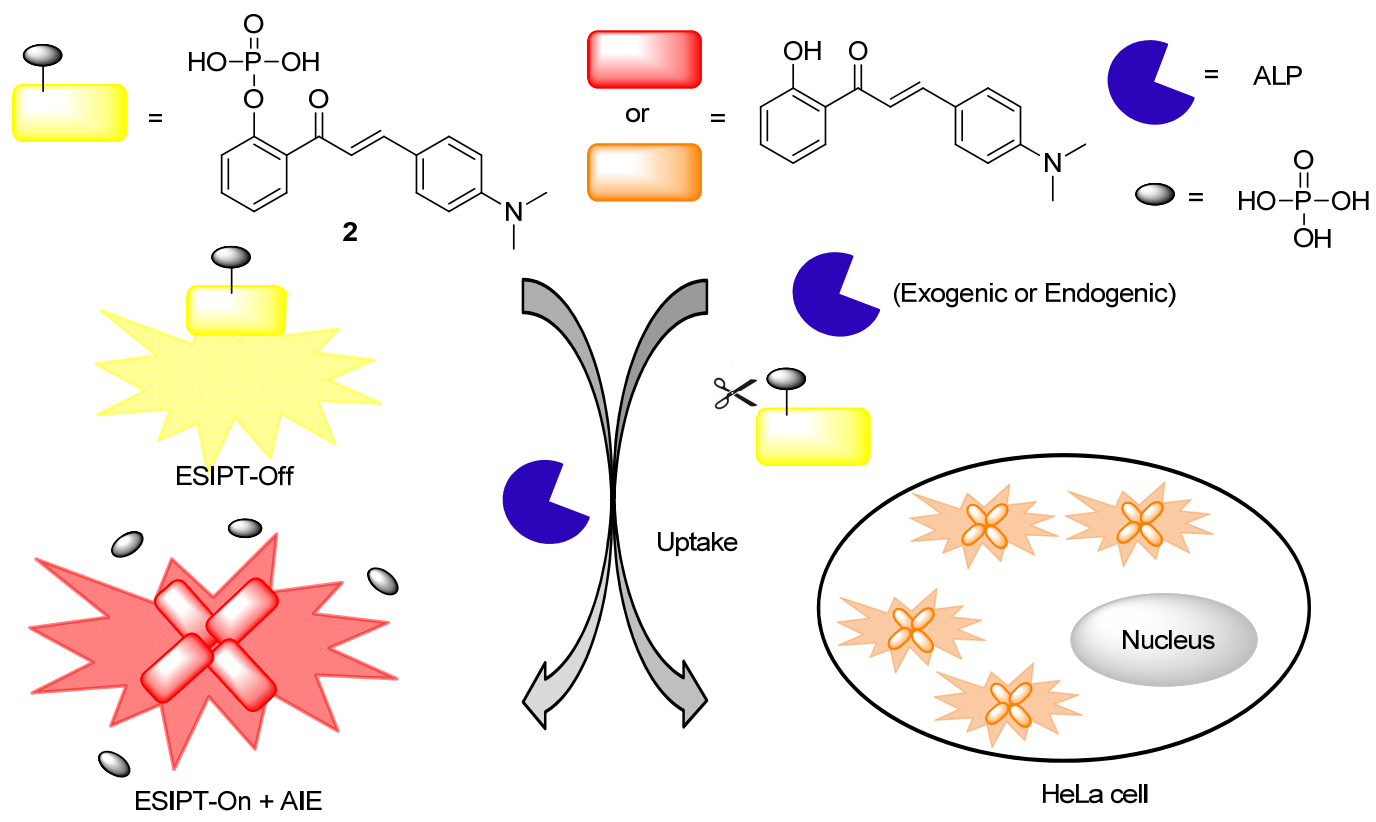

(Exogenic or Endogenic)

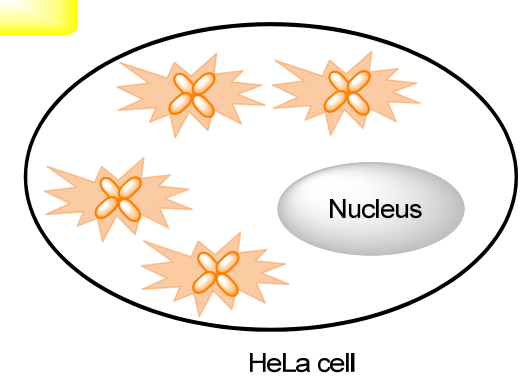

图式 1 探针 2 与 ALP 反应机理

Scheme 1 Mechanism of probe 2 reaction with ALP 
ALP 活性检测和成像研究. 其结构通过修饰的 2-差基查 尔酮得到. 该探针能溶于水, 并在缓冲溶液中发射出黄 绿色苂光. 在 ALP 存在的情况下, 由于酶切产物的形成 和聚集诱导效应的发生, 探针的荧光发射呈比率型苂光 响应, 探针体系逐渐由绿色变为深红色. 整个过程中荧 光的产生涉及激发态分子内质子转移和分子内的聚集 诱导发射机理, 其对 ALP 的活性范围为 $0 \sim 150 \mathrm{mU} / \mathrm{mL}$, 检测限为 $0.15 \mathrm{mU} / \mathrm{mL}$, 与同类结构相比较, 该结构检测 性能优良. 该探针具有良好的生物相容性, 可用于活细 胞 ALP 荧光成像研究. 此外, 该探针的红色荧光发射还 能抑制血清样品的自身荧光干扰, 并且还可用于细胞间 隙液中 ALP 的检测和相应活细胞的可视化研究. 该分 子设计策略为利用天然产物构建具有高灵敏度和聚集 态的比率型荧光探针提供了新的思路.

Kim 等 ${ }^{[18]}$ 基于调控的 ESIPT 过程, 设计合成了一种 能够快速定量分析 ALP 活性的苂光基质 3 (Eq. 2). 其检 测机理为荧光 turn-on 型 ESIPT 机制. 在 ALP 存在的水 溶液环境中, 苂光化合物表现显著的荧光发射信号. 该 探针对 ALP 具有极高的灵敏性, 其检测限为 $0.25 \mathrm{nmol} \cdot$ $\mathrm{L}^{-1}$. 不同寻常的是在 ALP 的苂光检测信号中出现了明 显的斯托克斯位移. 该化合物可以成功应用于实时检测 碱性磷酸酶的活性. 体外菼光试验研究结果将加快 ALP 抑制剂和催化剂的研发. 此外, 该工作有助于理解 ALP 在生命体系中的作用.

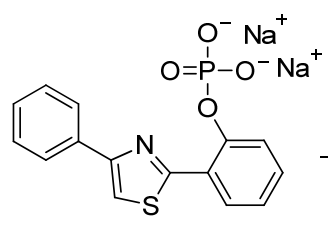

3

Soluble in water Weak blue fluorescent

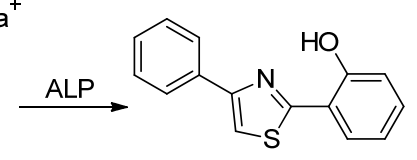

Insoluble in water Strong green fluorescent
接着该课题组 ${ }^{[18]}$ 设计合成了两种基于苯并噻唑结 构的荧光探针 4 和 $\mathbf{5}$, 用于活细胞中 ALP 的活性监测 (Scheme 2). 由于探针结构内部键旋转, 进而使荧光探 针 4 和 5 显示出微弱的苂光发射. 在 ALP 存在下, 探针 4 和 5 被 ALP 催化引起 $\mathrm{P}-\mathrm{O}$ 键的断裂, 随后酚氧基团 对腈基官能团的分子内亲核进攻导致亚氨基香豆素-苯 并噻唑的环化(4-a1), 这些环化产物不溶于水, 在激发 光下产生强的荧光发射可用于 ALP 检测. 该探针适用 于实时动态监测活体系统内源性 ALP 活性, 并可开发 ALP 抑制剂和激活剂, 进一步扩大了 ALP 检测苂光分 子工具箱, 有助于其在活体系统中的生物学和病理学的 研究.

韩克利等 ${ }^{[19]}$ 利用腺苷一磷酸 $(A M P) /$ 单磷酸鸟苷

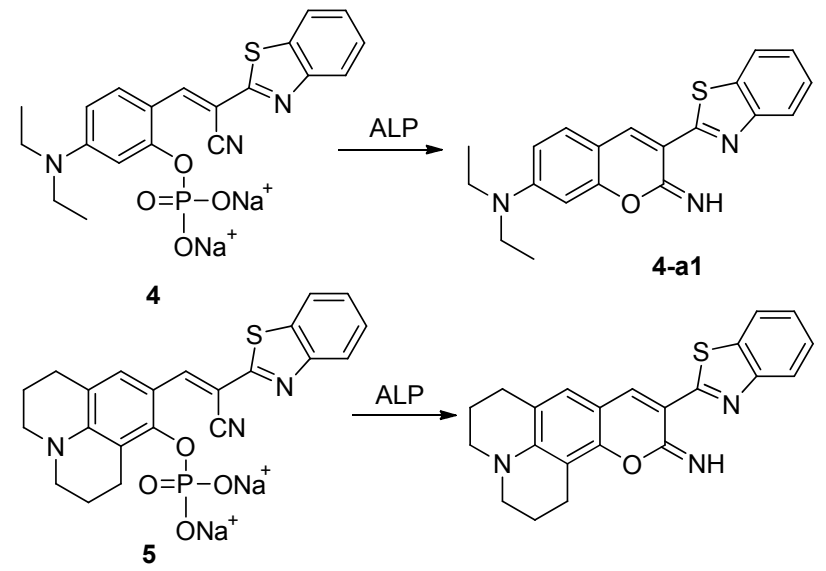

图式 2 探针 4 和 5 与 ALP 反应机理

Scheme 2 Reaction mechanism of probes $\mathbf{4}$ and $\mathbf{5}$ with ALP

(GMP)类似物的亲和性，合成了一种基于苯并噻唑结构 的 ESIPT 苂光探针 6 , 可以在水溶液和生物体内进行 ALP 的实时监测(Eq. 3). 在该结构设计中, 将 AMP 和 GMP 引入到基于 2-(2-羟基苯基)-苯并噻唑的探针中, 使得所合成探针具有高选择性、高亲和力和低毒性等特 点. 在水溶液中, 该探针无荧光发射, 当加入 ALP 后, 由于 ALP 的水解作用使磷酸二酯被水解，产生强的苂 光发射. 然而, 在 ALP 抑制剂的存在下，荧光发射强度 明显下降. 在整个识别过程中, AMP 和 GMP 被证明是 实现识别 ALP 酶所必需的物质. 该探针适用于生物系 统(如活细胞和斑马鱼模型)中内源性 ALP 活性的实时动 态监测.

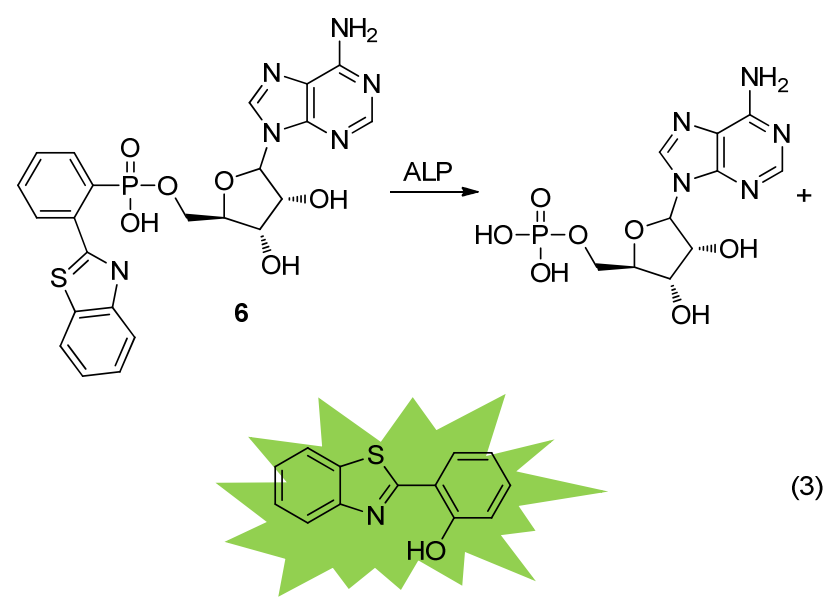

范春蕾等 ${ }^{[20]}$ 设计合成了一种基于 2-(苯并咪唑-2-酰 基)苯基磷酸的 ESIPT 比色苂光探针 7, 用于 ALP 苂光 检测 (Eq. 4). 其检测原理为 2-(2-羟基苯基)苯并咪唑 ( $\mathrm{HPBI})$ 的 ESIPT 过程, 通过羟基保护/脱保护反应进行 调节. HPBI 与 $\mathrm{POCl}_{3}$ 磷酸化后，由于 ESIPT 过程被阻止， 其在 $363 \mathrm{~nm}$ 处只有一个发射峰. 然而, ALP 在 Tris- $\mathrm{HCl}$ 缓冲液中发生选择性酶解, 探针 7 返回到 HPBI 结构, 开 
启 ESIPT 过程, 导致苂光强度在 $363 \mathrm{~nm}$ 处减弱, 而在 $430 \mathrm{~nm}$ 处的新的荧光峰左右升高. 且 430 和 $360 \mathrm{~nm}$ 处 的荧光强度比值 $\left(I_{430} / I_{360}\right)$ 随 ALP 活性的增加呈线性增 加, 最高可达 $0.050 \mathrm{U} / \mathrm{mL}$, 检出限为 $0.0013 \mathrm{U} / \mathrm{mL}$. 该探 针结构简单且灵敏度高, 可用于复杂样品的 ALP 分析 检测.

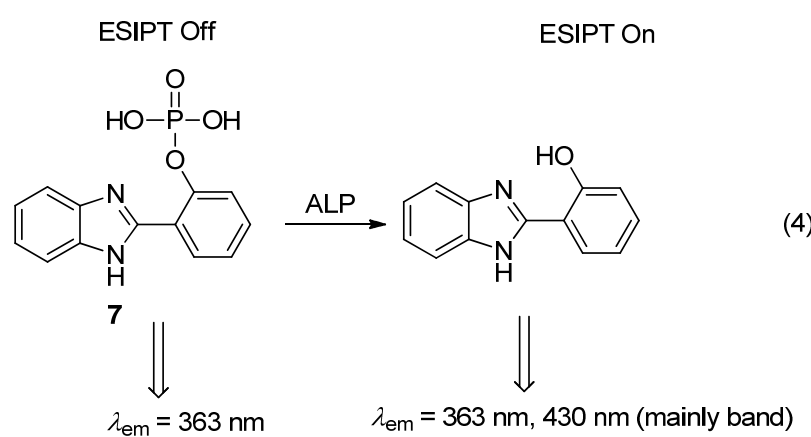

吴加胜等 ${ }^{[21]}$ 设计合成了一种基于分子内电荷转移 (ICT)特性的比率型荧光探针 8 , 用于 ALP 的检测(Eq. 5). 在 ALP 存在的条件下, 探针的苂光光谱中出现了从 550 到 $650 \mathrm{~nm}$ 明显的红移现象, 并产生比率的荧光信 号. 其苂光光谱的改变归因于探针的磷酸盐官能团裂解 和水解作用, 进而产生分子内的电荷转移. 其荧光强度 随浓度的变化在 50 200 U/L 的范围内呈线性关系, 且 呈比率型变化, 而其他竞争性生物物种不会引起明显的 荧光变化. 该探针具有较低的细胞毒性以及对 ALP 高 选择性, 可应用于活细胞中内生 ALP 荧光成像研究.
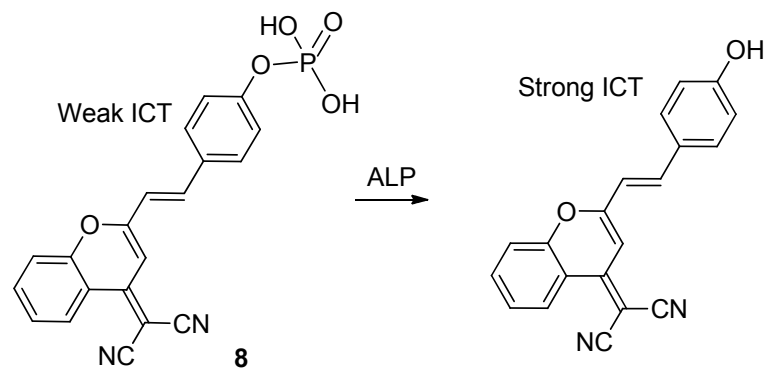

\section{2 基于聚集诱导发光的传感体系}

聚集诱导发光(aggregation-induced emission, AIE)机 制自从 2001 年由唐本忠课题组 ${ }^{[22,23]}$ 报道以来, 已被广 泛应用于各种离子和小分子的传感检测. 研究发现, AIE 分子在稀溶液时没有荧光发射, 在聚集状态时产生 强的荧光发射, 其具有与传统的有机小分子的聚集荧光 淬灭(aggregationcaused quenching, ACQ)效应相反的特 性. 其发光机理是由于聚集体的形成导致分子内的转动 被限制(restriction of intramolecular motions, RIM) ${ }^{[23]}$. 基 于 AIE 效应的生物荧光探针具有各种优势, 如低的苂光
背景、高的信噪比和优异的抵抗光漂白性. 在诸多被开 发的 AIE 分子中, 四苯乙烯结构(tetraphenylethene, TPE) 因其发光性能优良及容易修饰合成等优点作为 ALP 荧 光传感器的信号报告基团.

唐本忠等 ${ }^{[24]}$ 设计合成了一种基于 TPE 苂光团的聚 集诱导发光苂光探针 9, 其能够应用于 ALP 的检测和其 酶活性的评价(Eq. 6). 其检测机理是探针与 ALP 的特异 性反应(即利用 ALP 对含磷酸官能团的 TPE 结构进行脱 磷酸化作用, 使产物分子内的旋转被限制). 该探针具 有良好的水溶性, 在水溶液中几乎无荧光发射, 在 ALP 存在情况下，通过酶水解作用使磷酸基团裂解，产物发 生 AIE 效应表现出强的苂光. 在 Tris 缓冲溶液中, 该探 针对 ALP 具有高灵敏性和选择性, 其检测限为 11.4 $\mathrm{pmol} \cdot \mathrm{L}^{-1}$ 或 $0.2 \mathrm{U} / \mathrm{L}$, 在 $3 \sim 526 \mathrm{U} / \mathrm{L}$ 范围内, 具有很好的 线性关系. 该探针被成功应用于血清中 ALP 的检测, 其 线性范围为 $30 \sim 135 \mathrm{U} / \mathrm{L}$. 此外, 该探针被成功应用于 血清中 ALP 的检测, 表明其具有检测实际样品中 ALP 水平的潜在可能. 该研究工作提出的策略可以推广到构 建其他酶的分子探针的设计中, 为其它苂光探针的开发 提供新的思路.
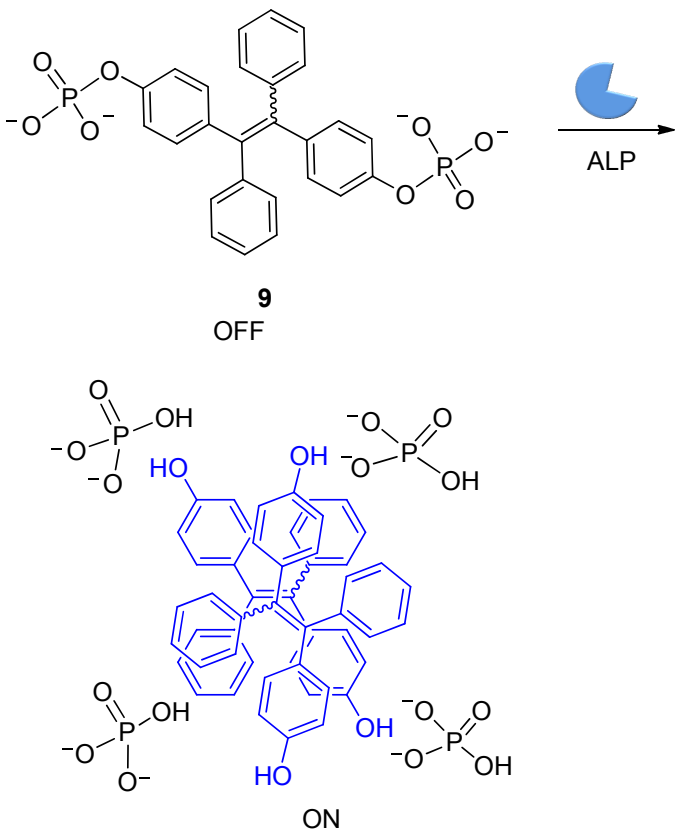

张德清等 ${ }^{[25]}$ 报道了一种甲氧基修饰的基于 TPE 结 构的 AIE 苂光化合物 10, 用于 ALP 活性检测和抑制剂 筛选试验，此试验方法可以测定低至 $18 \mathrm{mU} / \mathrm{mL}$ 的 ALP 浓度 (Scheme 3). 化合物 10 可用于在细胞环境中 ALP 检测, 化合物能够进入细胞, 且当 ALP 存在时, 产生绿 色的苂光. 其测定 ALP 的原理为荧光化合物在 ALP 存 在下，通过脱磷酸化作用水解成溶解度低和容易聚集的 TPE 衍生物结构. 与其它报道的 ALP 测定方法相比，该 
苂光检测体系具有试验可在纯水溶液中进行、响应时间 较短、适用于细胞中 ALP 的测定及化合物易于制备等 优点. 因此, 该 ALP 检测方法具有较强的实用性.

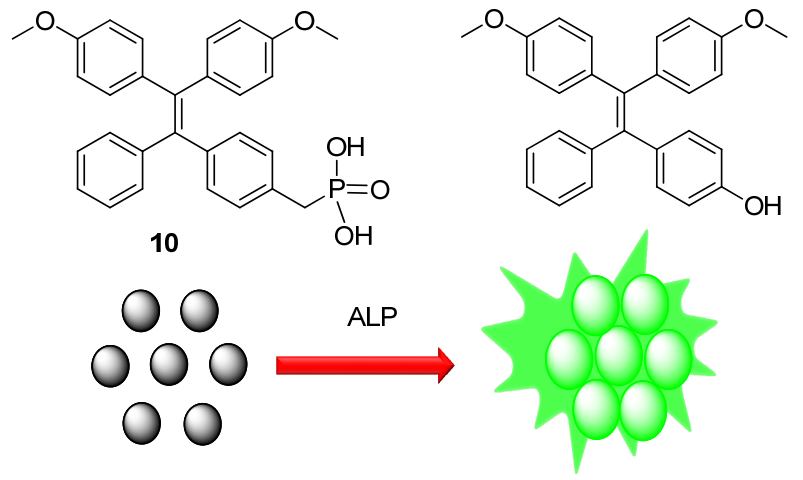

图式 3 探针 $\mathbf{1 0}$ 与 ALP 反应机理

Scheme 3 Mechanism of probe $\mathbf{1 0}$ reaction with ALP

李新明等 ${ }^{[26]}$ 开发了一种 TPE 分子结合酪氨酸-磷酸 盐的 AIE 荧光探针 11 (Eq. 7). 在该探针结构中, 硌氨 酸-磷酸盐和 TPE 结合生成新的两亲分子, 由于分子结 构中含有两个磷酸基团, 其在 $\mathrm{pH}=7.4$ 的水溶液环境中 表现出良好的溶解性. 在 ALP 存在的条件下, 发生脱磷 酸化反应产生强的苂光发射信号来检测 ALP 的活性. 此外, 探针在 $\mathrm{pH}=2.5$ 的水溶液中, 通过自组装形成新 颖的超分子水凝胶, 其荧光发射强度受到 $\mathrm{pH}$ 变化的影 响. 另外, 两亲分子自组装形成高度有序的胶束纳米结 构作为一种有效的模板促进成核现象和磷酸钻的生长. 氨基酸和多肽是一类重要的生物活性物质, 其具有生物 分子识别和超分子自组装的能力, 使得该探针在氨基酸 和多肽方面具有广泛的应用前景. 该研究结果表明用

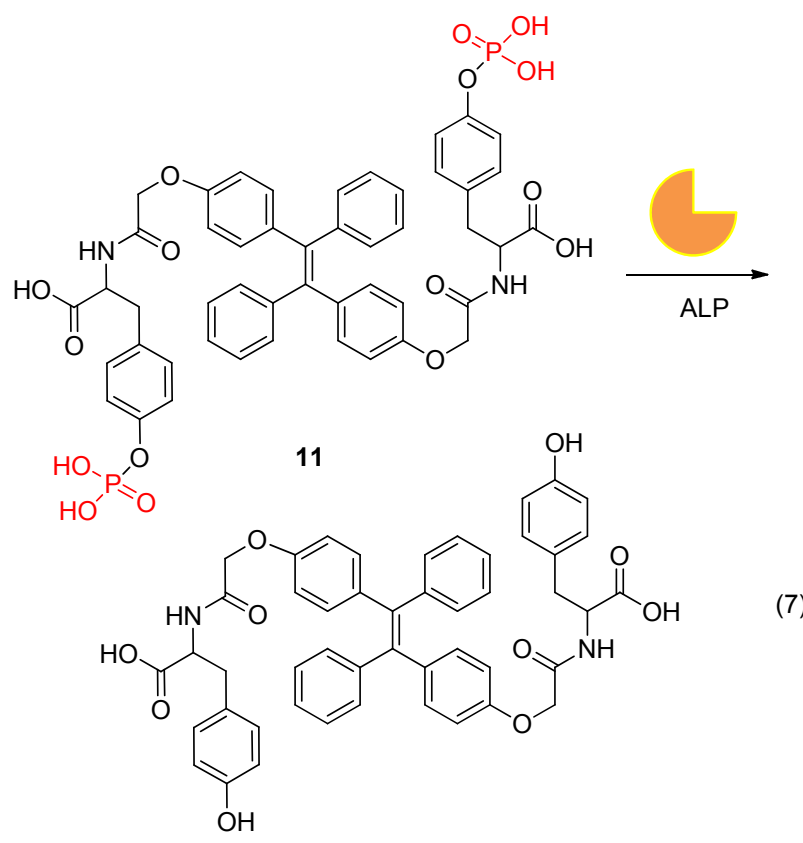

TPE 和氨基酸结合形成的分子结构具有潜在的多功能 性质, 包括酶活性测定, 细胞成像或药物传递等.

吴水珠等 ${ }^{[27]}$ 设计合成了一种基于 TPE 结构的苂光 探针 12, 用于 ALP 的检测及细胞成像 (Scheme 4). 该结 构中，强吸电子的氰基处于化合物结构的中间位置，亲 水性的磷酸基识别基团处于结构的末端位置. 该供体受体-受体(D-A-A)型结构与 TPE 结构相比较具有长的 激发和发射波长，同时，增加共轭长度时保持良好的水 溶性. 在水溶液中, 探针对 ALP 具有较高的灵敏度. 在 ALP 存在的条件下, 磷酸盐基团被水解, 结构 TPE-CN-OH 产生聚集诱导效应导致水溶性降低, 并产 生绿色苂光，从而实现 ALP 的检测. 该探针具有良好的 生物相容性, 能够成功应用于细胞成像研究. 其设计方 法为其他酶检测策略提供了新的思路.

韩宝航等 ${ }^{[28]}$ 设计合成了一种基于 AIE 效应的新型 阳离子水溶性 TPE 衍生物荧光探针 13 (Scheme 5). 该探 针是将氨基葡萄糖盐酸盐嫁接到 TPE 结构上，不仅可以 提高其水溶性和生物相容性, 而且为其它人工或天然聚 阴离子之间的静电相互作用提供了许多带正电荷氨基 结合位点. 利用聚集诱导增强发光特性的优势, 开发出 了这种便捷的检测 ALP 的方法. 该探针聚集络合物与 十二烷基磷酸单酯能够产生 turn-on 的光致发光现象. 当 ALP 存在时, 探针的苂光逐渐降低, 其原因为酶引发 的探针与十二烷基磷酸单酯聚集被破坏. 该研究在生物 大分子的检测及相关抑制剂篮选方面具有广泛的应用 前景.

最近，赵娜等 ${ }^{[29]}$ 利用邻羟苄基与 ALP 的反应设计 了一种具有 AIE 效应的苂光探针 14, 其对 ALP 表现出 高灵敏性，可以用于检测 ALP 和监测其酶活性(Scheme 6). 探针结构由磷酸基团和四苯乙烯衍生物组成. 其在 水溶液中的表现出良好的溶解性，显示出较弱的荧光发 射. 在 ALP 存在的情况下，探针通过脱磷酸化作用释放 出 TPE 水解产物, 使荧光发射显著增强. 该探针对 ALP 的检测限低至 $0.0077 \mathrm{U} / \mathrm{L}$, 溶液中线性范围为 $0 \sim 30$ $\mathrm{mU} / \mathrm{mL}$. 其在稀释的血清样品中具有优异的适用性，能 够在血清样品中高灵敏度地检测 ALP 及其活性，证明 了其在临床诊断和生物医学研究中的潜在应用价值. 此 外，该探针能够应用于 ALP 活性测定和 ALP 抑制剂篮 选方面的研究.

\section{3 基于降低给电子能力(D d-E)或扰乱共轭 $\pi$ 电子的传感体系 $(D \pi-C)$}

由于近红外(NIR)苂光传感器具有长的激发和发射 波长(波长大于 $650 \mathrm{~nm}$ ), 因此有较深的组织穿透性. 此 外其具有低能量、弱自苂光背景干扰、良好的光稳定性 


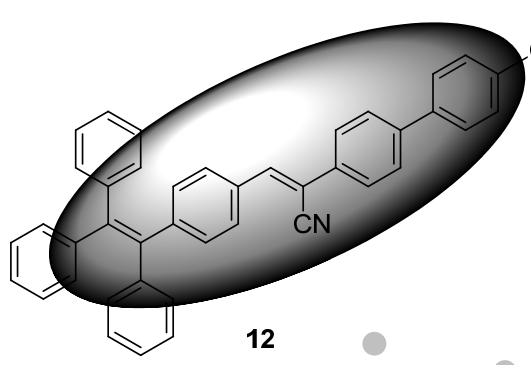

0

Solution weak fluorescence

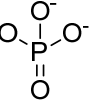
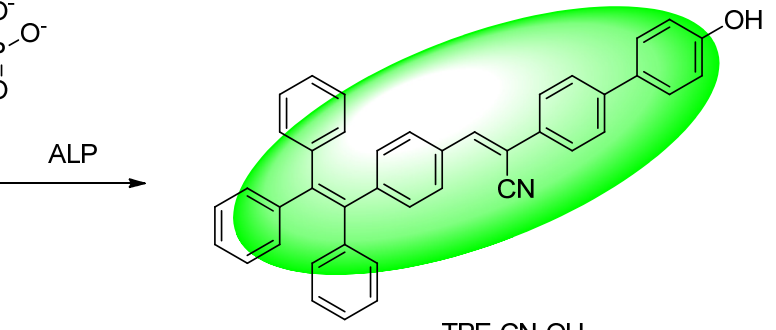

TPE-CN-OH

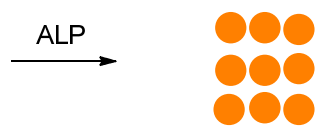

Aggregation weak fluorescence

图式 4 探针 12 与 ALP 反应机理

Scheme 4 Mechanism of probe 12 reaction with ALP

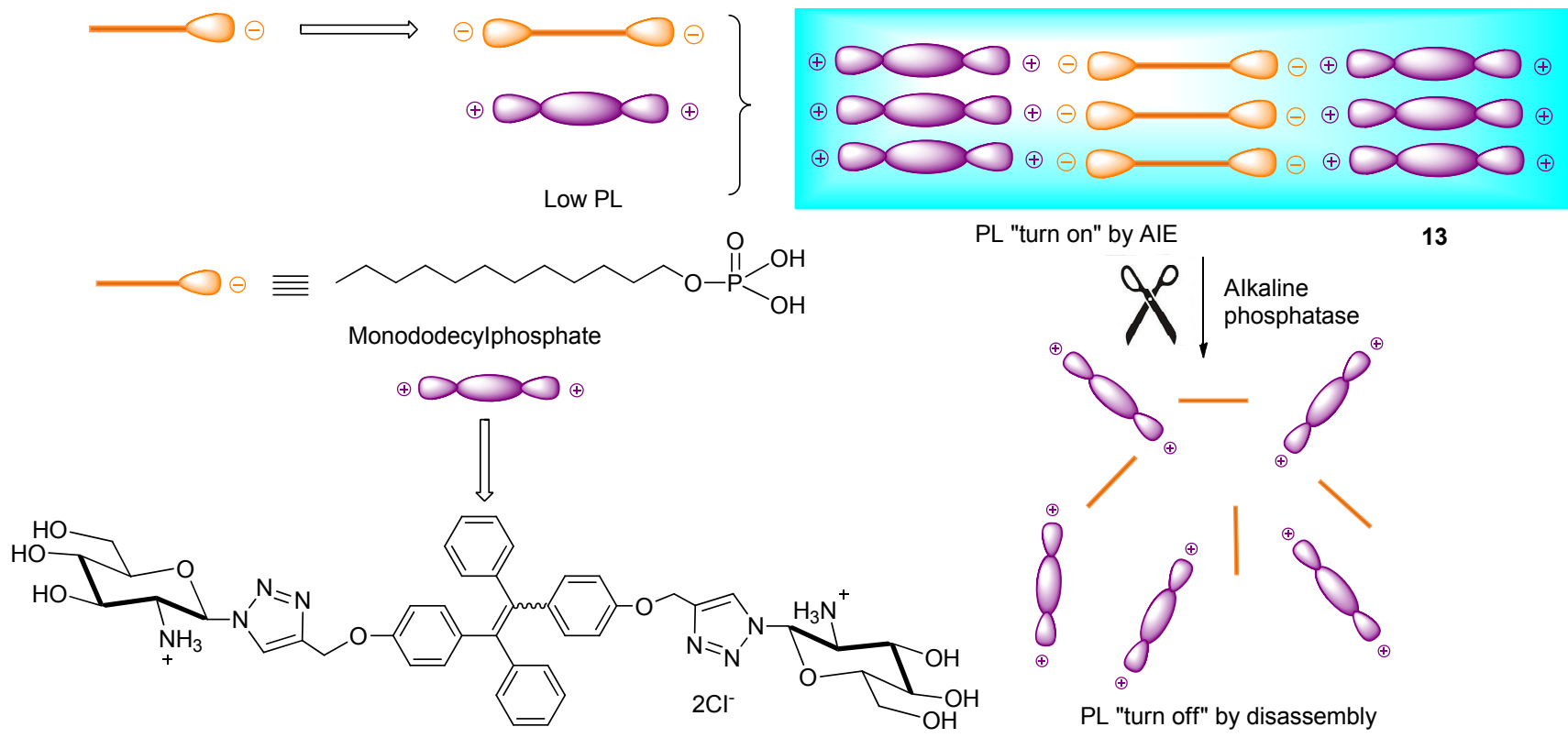

图式 5 探针 $\mathbf{1 3}$ 与 ALP 反应机理

Scheme 5 Mechanism of probe 13 reaction with ALP

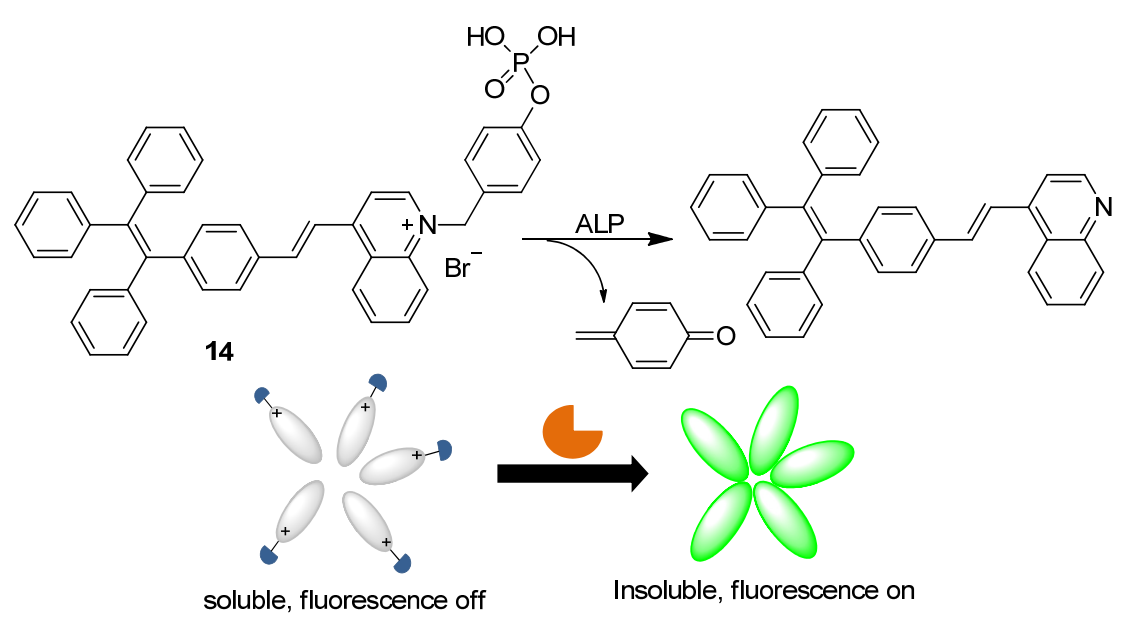

图式 6 探针 14 与 ALP 反应机理

Scheme 6 Mechanism of probe 14 reaction with ALP 
和对生物样品低的损伤性等特点 ${ }^{[30]}$. 基于以上一些特 点, 近红外菼光传感器极大地促进了体内生理过程的成 像研究, 因此其在生物医学领域具有很大需求. 其中, 半花菁结构是理想的近红外成像苂光团, 已被用作 ALP 传感体系中的信号报告基团. 其检测机理主要是降低化 合物的给电子能力(Diminish the electron donating ability, D d-E) 或扰乱共轭 $\pi$ 电子体系 (Disruption of the $\pi$-Conjugated Systems, D- $\pi$ C). 然而, 该类 ALP 苂光传 感体系的研究报道稀少.

半花菁结构是传统的近红外苂光染料, 具有高的量 子产率、光稳定性以及良好的活体成像性能等, 适合于 近红外苂光传感器的构筑. 孙红燕等 ${ }^{[31]}$ 利用 ALP 与磷 酸酶反应的特点设计了一种 ALP 近红外苂光探针 15, 用于检测体内体外 ALP 的活性(Eq. 8). 其结构包含一个 半花菁苂光团和一个磷酸酶反应位点. 该探针对 ALP 表现出高灵敏性, 当 ALP 存在时, 其荧光发射增强 66 倍, 检测限为 $0.07 \mathrm{U} / \mathrm{L}$, 低于大多数先前报道的 ALP 苂 光探针. 此外, 该探针具有高的反应活性、良好的水溶 性、强组织穿透能力及弱背景干扰等优势, 特别是探针 具有在长波长区域产生荧光发射的特性, 使其成为细胞 及生物组织成像的理想候选者. 进一步研究发现, 该探 针能够应用于细胞和小鼠中 ALP 活性的检测, 具有良 好的生物相容性和细胞内成像性能. 该探针为生物组织 中 ALP 活性的检测提供有效的方法.

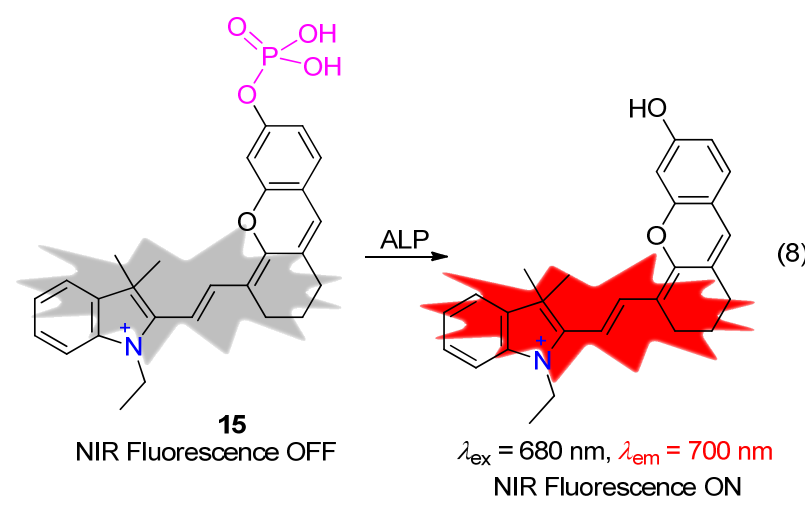

与上述工作同时，李春艳等 ${ }^{[32]}$ 设计了一种类似结 构的苯并半花菁近红外苂光探针 16, 用于检测内源性 ALP 的活性(Eq. 9). 其荧光变化的机理是在 ALP 催化作 用下，使得探针结构中的磷酸基团被䢃裂，实现苂光信 号从 turn-off 到 turn-on 的转变. 其荧光发射峰在近红外 区 $738 \mathrm{~nm}$. 该探针具有结构简单、易合成且在近红外区 能发光等特点. 其在 $738 \mathrm{~nm}$ 产生近红外苂光发射, 适用 于活体生物成像. 该近红外探针在 ALP 的识别性能方 面实现了显著提升, 并对 ALP 具有高的灵敏性, 探针检 测限为 $0.003 \mathrm{U} / \mathrm{mL}$. 该研究工作实现了各种生物样本中
内源性 ALP 活性的近红外苂光检测.

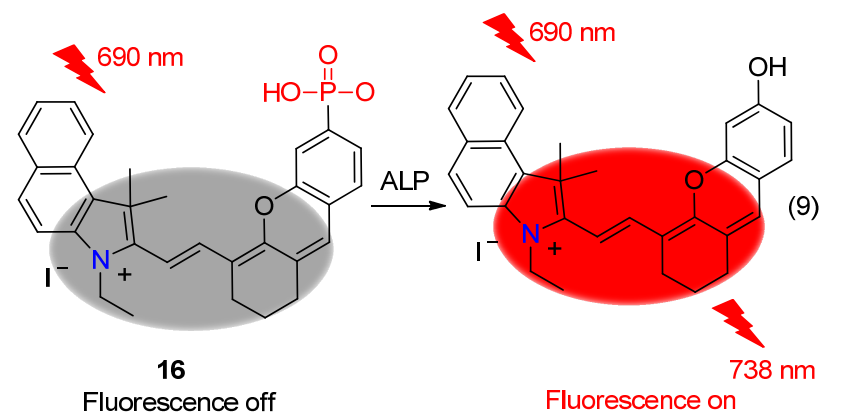

最近，张鹏等 ${ }^{[33]}$ 报道了一种基于花菁骨架的比率 型近红外 ALP 荧光探针 17. 在结构设计中, 作者将掩蔽 剂引入到内消旋氧原子的花菁骨架, 用于调节花菁染料 共轭 $\pi$ 电子体系, 磷酸基团作为 ALP 水解催化的官能团 (Eq. 10). 这种具有高选择性和高灵敏性的比率型 ALP 苂光探针的设计策略通过光谱研究和生物成像研究得 到了证明. ALP 存在的情况下，探针具有高的灵敏性和 选择性、响应时间短和低苂光背景的特点，其最低检测 限为 $0.16 \mathrm{mU} \cdot \mathrm{mL}^{-1}$. 该探针可以成功应用于生物细胞 中 ALP 的检测, 并具有高的光响应活性. 该研究结果对 探索生物体系中 ALP 的生理功能具有较大潜在应用价 值.
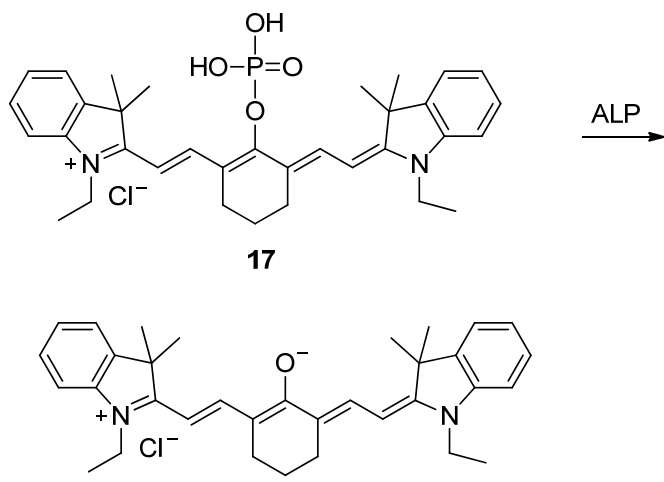

王兴华等 ${ }^{[34]}$ 开发了一种能够在生物器官中监测 ALP 的近红外苂光探针 18. 该苂光分子具有长的发射 波长, 且对 ALP 具有高灵敏性和高选择性(Eq. 11). 在 ALP 存在的情况下, 探针在 $723 \mathrm{~nm}$ 发射波长处表现出 56 倍的苂光信号增强, 其检测限为 $0.042 \mathrm{U} \cdot \mathrm{L}^{-1}$. 该探针 被成功应用于不同类型细胞内生和外生的 ALP 水平的 测定. 该研究结果确定该探针在肝脏和骨骼的临床诊断 中具有潜在的应用价值.

于法标等 ${ }^{[35]}$ 设计合成了一种基于对称半花菁结构 的近红外苂光探针 19, 用于检测不同细胞系和小鼠肿 瘤模型中 ALP 的水平(Scheme 7). 在该探针结构中, 七 甲川花青素结构作为苂光调节器, 磷酸盐单酯作为 ALP 酶催化响应部分. 其荧光检测机理为 $\pi$ 电子共轭体系, 


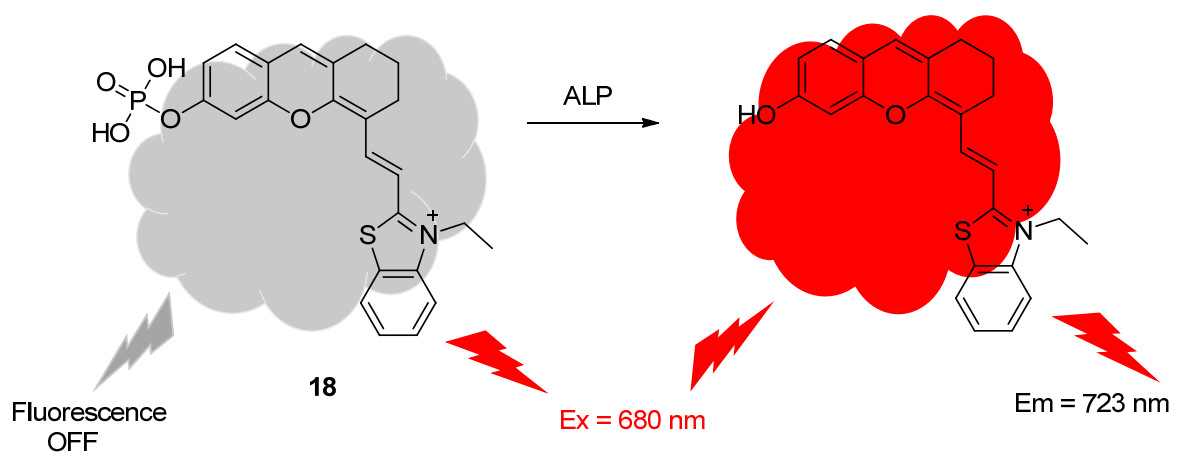

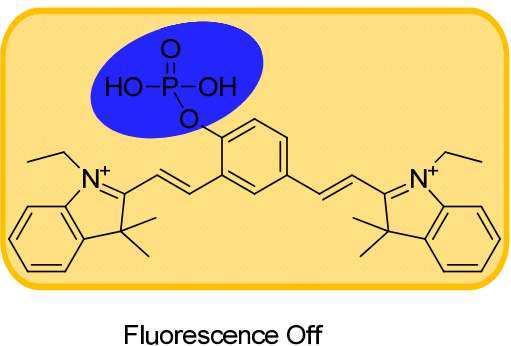

19

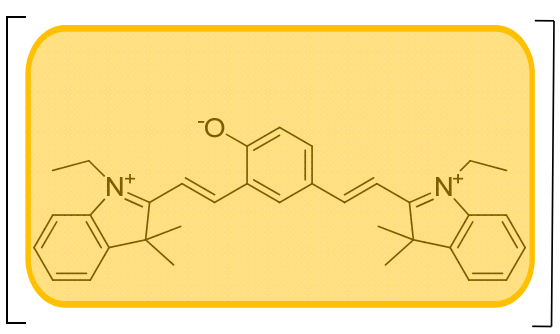

Fluorescence Off

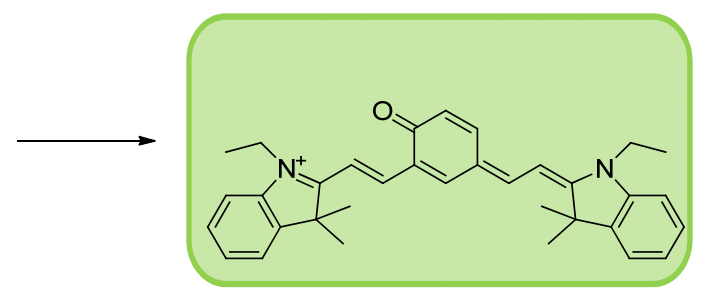

Fluorescence On

图式 7 探针 19 与 ALP 反应机理

Scheme 7 Mechanism of probe 19 reaction with ALP

通过 ALP 催化磷酸盐单酯基团裂解引发 off-on 苂光转 变. 该探针对 ALP 表现出高的选择性和较好的灵敏度, 不但可以识别不同细胞系中 ALP 水平, 而且可以区分 小鼠肝肿瘤模型和正常小鼠体内 ALP 水平的变化. 在 不存在其他酶、离子和蛋白相互作用的情况下, 对 ALP 有较好的选择性和敏感性. 该探针分子为揭示 ALP 在 生理和病理过程中的作用提供了可能, 具有潜在的实用 性.

\section{4 基于荧光内滤效应(IFE)的传感体系}

功能纳米材料因可以混合多种成分, 表现出优异的 光学、电学、磁性和生物活性等性能, 如今成为新材料 研究领域的热点课题 ${ }^{[36]}$. 到目前为止, 纳米材料在大 小、形状及可控合成方面已经得到很好的发展. 研究发 现, 纳米材料的尺寸和形状相关性质在高性能传感器的 制备中尤为重要. 此外, 纳米材料通常具有高的表面能 量和相对体积比例, 从而可以捕获更多的标记目标且具 有较高灵敏度. 其中, 量子点材料和金属纳米粒子已作
为 ALP 传感体系中的载体或信号报告基团. 苂光内滤 效应(IFE) 是由于荧光物质的吸收光谱与激发或发射光 谱重叠时, 导致检测系统吸收激发光或发射光的现象. IFE 效应通过将分析吸收信号转换为荧光信号, 如今已 经成为设计和开发新型传感器的一种有效策略.

吴永宁等 ${ }^{\left[{ }^{[7]}\right.}$ 设计合成了一种基于 IFE 效应的探针 20, 用于 ALP 的检测(Scheme 8). 在合成过程中, 采用 一锅合成法以高达 $49 \%$ 的量子产率制备了 $N$-掺杂碳量 子点 (CDs), 并将其作为 IFE 的荧光团. 利用对硝基苯磷 酸盐(PNPP)作为 ALP 底物, 其酶催化产物(对硝基苯酚) 具有强大的吸附作用, 影响荧光团(CDs) 的激发. 在 ALP 存在的条件下, PNPP 被转化对硝基苯酚, 诱导吸收 带从 $310 \mathrm{~nm}$ 过渡到 $405 \mathrm{~nm}$, 导致对硝基苯酚的吸收与 $\mathrm{CDs}$ 的激发互补重叠. 由于竞争性吸收的存在, CDs 的 苂光激发明显减弱, 最终导致 CDs 的荧光淬灭. 该探针 在 $0.01 \sim 20 \mathrm{U} / \mathrm{L}$ 范围内有良好的线性关系 $\left(R^{2}=0.996\right)$, 检测限为 $0.001 \mathrm{U} / \mathrm{L}$ (信噪比为 3). 该方法可用于血清中 ALP 的检测和细胞成像研究. 该研究结果提出的基于 
IFE 的 CDs 苂光传感策略, 由于不需要受体与荧光团之 间的结构修饰或连接, 为酶活性检测的苂光探针的发展 提供了新的思路.

A
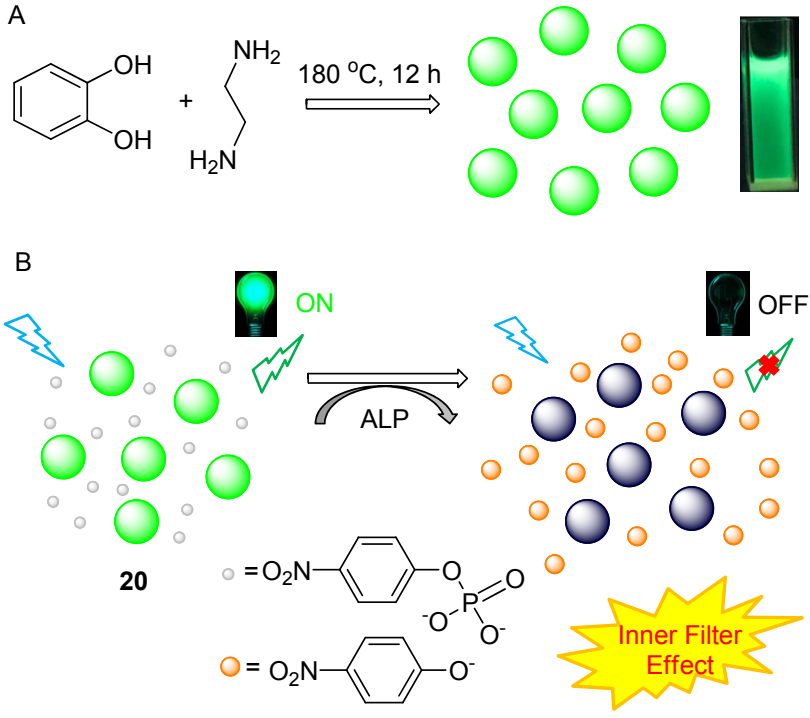

图式 8 探针 $\mathbf{2 0}$ 与 ALP 反应机理

Scheme 8 Mechanism of probe 20 reaction with ALP

最近，何治柯等 ${ }^{[38]}$ 采用简单有效的方法开发了基 于对硝基苯酚 $\mathrm{CdTe} / \mathrm{CdS}$ 的苂光量子点 ALP 苂光探针 21, 其具有明显的 IFE 效应(Scheme 9). 在探针 CdTe/

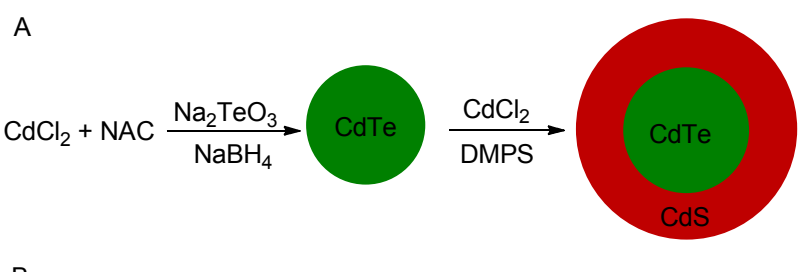

B

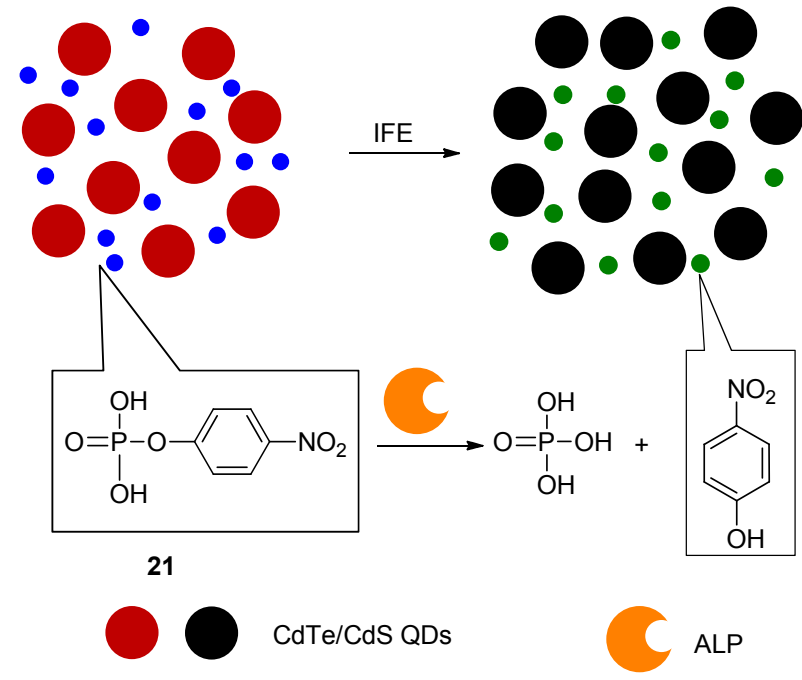

图式 9 探针 21 与 ALP 反应机理

Scheme 9 Mechanism of probe 21 reaction with ALP
$\mathrm{CdS}$ QDs 的制备过程中，采用 $\mathrm{Na}_{2} \mathrm{TeO}_{3}$ 为 $\mathrm{Te}$ 源，以二颈 基化合物为 $\mathrm{S}$ 源和表面配体. 该苂光探针具有良好的苂 光性能、高量子产率(80\%)以及化学/光稳定性. 基于对 硝基苯酚磷酸盐在 ALP 催化作用下的能够水解的特点, 可以有效地淬灭量子点的荧光. 因此, 可以用于 ALP 的 检测，其在 $2.2 \sim 220 \mathrm{U} / \mathrm{L}$ 的浓度范围内具有良好的线性 关系, 检出限低至 $0.34 \mathrm{U} / \mathrm{L}$. 该方法成功应用于人血清 中 ALP 的化验. 由于 QDs 具有宽的吸收, CdTe/CdS QDs 可以广泛应用于 IFE 化学或生物传感器中.

任雪琴等 ${ }^{[39]}$ 设计合成了一种基于对硝基苯基磷酸 盐(PNPP)的金纳米团簇(AuNCs)IFE 传感器 22, 其可以 用于检测 ALP (Scheme 10). 采用一锅法合成，其量子产 率为 $12 \%$, 可以直接作为苂光材料应用. 当 AuNCs 与 PNPP 混合时, 由于 PNPP 的吸收光谱与 AuNCs 的激发 光谱有重叠, 使得 AuNCs 的苂光内滤效应减弱进而发 生明显荧光淬灭. 而在 ALP 存在下, PNPP 被催化水解 成对硝基苯酚，从而使 AuNCs 苂光得到恢复. 这种新型 的 turn-on ALP 苂光探针, 其检测限可低至 $0.002 \mathrm{U} / \mathrm{L}$ (信 噪比为 3). 并且具有高灵敏度、高选择性、低背景信号 及高信号输出等优点. 此外, 该探针被成功应用于血清 ALP 抑制剂的检测和血清 ALP 的测定. 在 $0.02 \sim 50 \mathrm{U} / \mathrm{L}$ 范围内有良好的线性关系. 这种非常简单的制备传感器 方法将促进纳米化学和生物探针的发展.

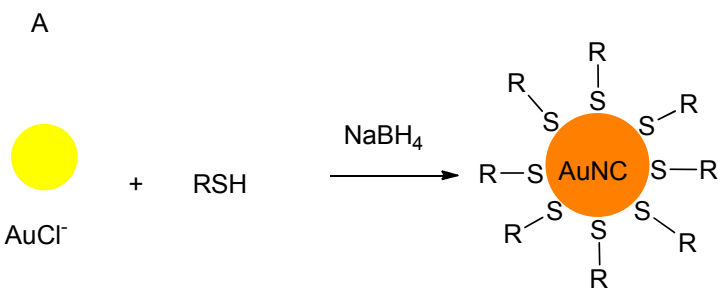<smiles>O=C(O)CCCCCCCCCCS</smiles>

B
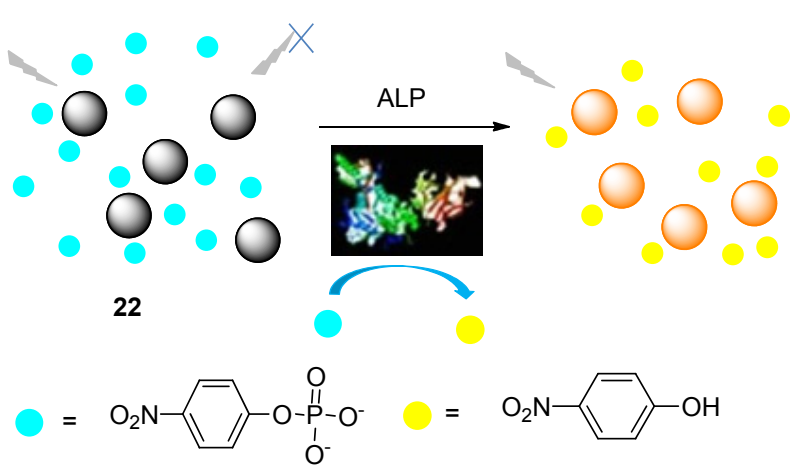

图式 10 探针 22 与 ALP 反应机理

Scheme 10 Mechanism of probe 22 reaction with ALP 


\section{5 其它类型的传感体系}

梁高林等 ${ }^{[00]}$ 利用溶胶-凝胶转变与荧光淬灭过程, 制备了一种定量检测体外和活细胞中 ALP 活性的荧光 探针 23 (Eq. 12). 在 ALP 催化作用下, 探针自组装成纳 米纤维, 进一步形成凝胶. 伴随该溶胶-凝胶转变过程, 探针荧光发生淬灭. 该检测方法能够检测 $0 \sim 2.8 \mathrm{U} / \mathrm{mL}$ 范围内的 ALP 活性, 检测限(LOD)为 $0.06 \mathrm{U} / \mathrm{mL}$. 此外, 通过 ALP 抑制剂处理的细胞成像试验研究发现, 探针 能够在细胞中检测 ALP 活性. 该方法可用于实时和定 量检测体外甚至活细胞中的 ALP 活性, 为 ALP 活性的 实时与定量检测提供了一种新的方法.

最近，孔金明等 ${ }^{[41]}$ 设计合成了一种基于电荷转移 诱导荧光淬灭的 ALP 活性苂光探针 24, 该探针核心结 构为铜配合物 $\mathrm{Cu}(\mathrm{BCDS})_{2}^{2-}$ (Scheme 11). 在 ALP 存在下,
底物抗坏血酸 2-磷酸水解释放出坏血酸(AA). 随后, 在 $\mathrm{AA}$ 介质中存在的 $\mathrm{Cu}(\mathrm{BCDS})_{2}^{2-}$ 被还原, 在 $402 \mathrm{~nm}$ 波长处 呈现出强烈的光致发光带, 导致探针的苂光由于电荷的 转移而被静态淬灭, 通过理论计算验证了苂光淬灭的机 理. 该探针具有较大的斯托克斯位移 $(86 \mathrm{~nm})$, 对光漂白 具有很强的免疫能力. 该方法的优势在于不需要复杂的 探针设计即可以实现 ALP 活性的实时检测.

李新明等 ${ }^{[42]}$ 设计合成了一种基于磷酸盐和对羟基 苯衍生物反应的苂光探针 25 (Scheme 12). 该探针由磷 酸基团、对羟基苯衍生物以及苂光基团组成. 在 ALP 存 在的条件下, 探针上的磷酸部分在 ALP 催化下发生脱 磷酸化反应，通过 1,6-消除反应对羟基苯衍生物发生自 分解反应并释放适量的试卤灵(9-羟基-3-异吩噁嗪酮), 导致溶液由橙色变为紫色, 在 $585 \mathrm{~nm}$ 激发光照射下产

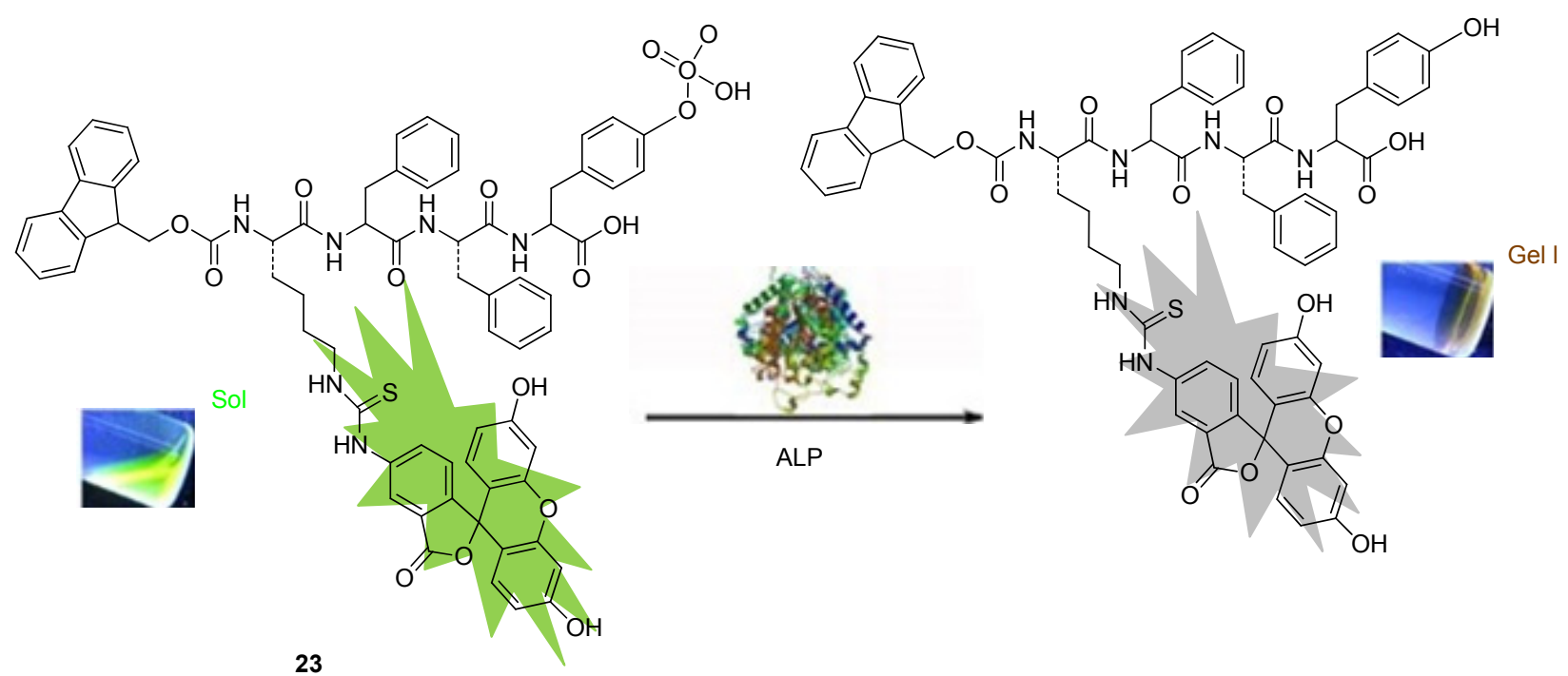

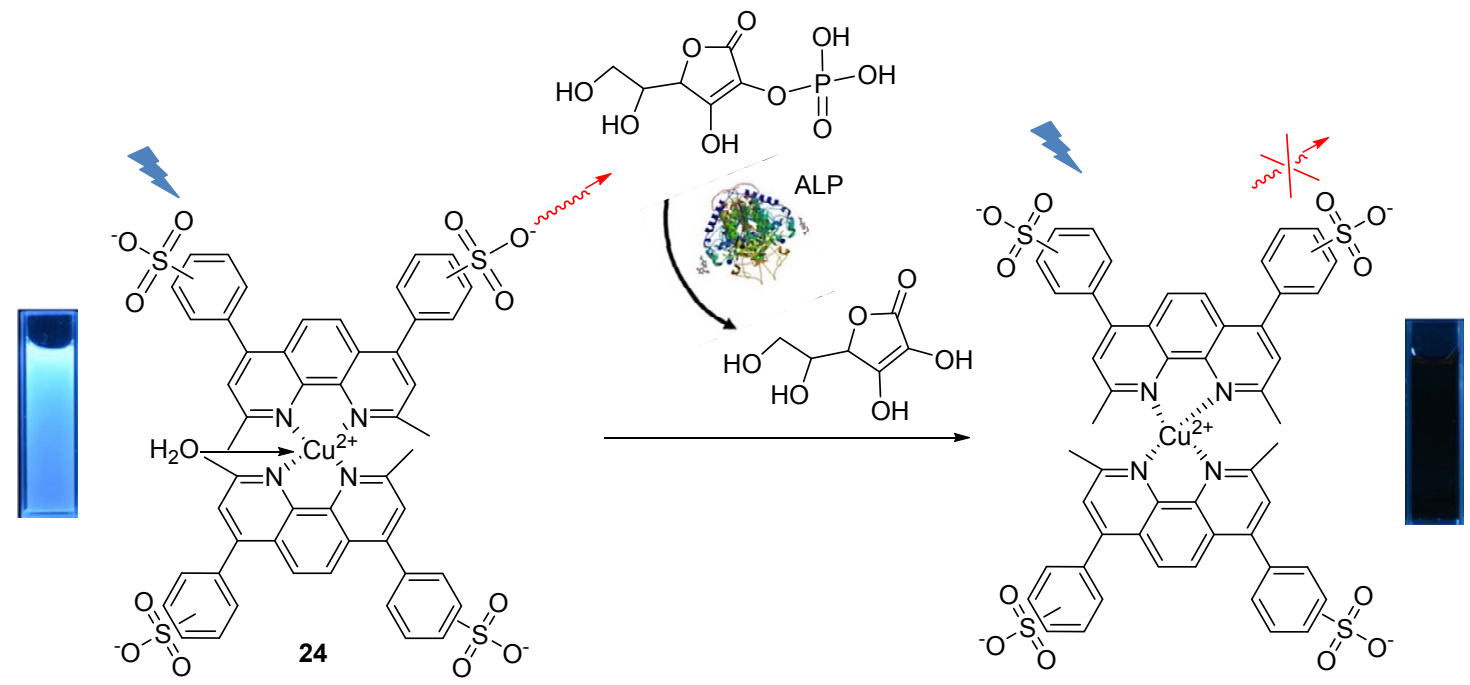

图式 11 探针 24 与 ALP 反应机理

Scheme 11 Mechanism of probe 24 reaction with ALP 
生强苂光发射, 与分析物相互作用后, 可通过肉眼观察 到颜色的改变并容易通过菼光光谱检测. 另一方面, 磷 酸盐部分与试卤灵之间的自组装连接可以降低空间位 阻, 提高探针对 ALP 的脱磷酸化反应. 二甲醌残留物可 以通过大多数介质(如 $\mathrm{H}_{2} \mathrm{O}$ ) 或细胞内靠近反应位点的其 它亲核试剂淬灭. 该探针可以通过裸眼和苂光检测的手 段对溶液中 ALP 实现快速、灵敏的检测, 检测限可低至 $1.09 \mathrm{U} / \mathrm{L}$, 并且具有良好的生物相容性以及实时监测活 细胞内源性磷酸酶活性的潜力.

于聪等 ${ }^{[43]}$ 利用季铵阳离子合成了一种基于菲四甲 酸二酐结构的荧光探针 26, 其可以用于实时检测 ALP 的活性以及抑制剂的笁选(Scheme 13). 当溶液体系中 存在三磷酸腺苷(ATP)时, 探针苂光被淬灭. 在 ALP 存 在的条件下, ATP 或探针复合物被解离, 探针荧光发射 被恢复, 而当加入 ALP 抑制剂后荧光发射下降. 该探针 基于荧光 turn-on 模式，可大大降低与苂光 “淬灭” 检测 相关的假阳性信号的干扰. 其次, 该检测体系为快速、 灵敏地检测 ALP 活性和抑制剂篮选提供了一种方便的 “mix-and-detect”方法. 利用普通分光光度计可以实时监
测荧光强度的变化. 此外，该探针易于制备，且底物 ATP 具有商业可利用性, 具有较高的经济效益.

曾钫等 ${ }^{[44]}$ 采用类似识别官能团，合成了一种比率 型荧光探针 27, 用于追踪 ALP 在体内的变化情况(Eq. 13). 该探针结构包含一个磷酸基团和一个胺 $-N$-氧化物 基团取代的 1,8-萗酰亚胺衍生物. 在 ALP 存在条件下, 1,8-菜酰亚胺衍生物中的磷酸基团被裂解，导致探针的 荧光光谱发生显著改变, 从而实现对 ALP 的荧光检测. 胺- $N$-氧化物基团的掺入使探针具有良好的水溶性和生 物相容性，在水介质中实现对 ALP 的比率检测。该探针 检测限为 $0.38 \mathrm{U} / \mathrm{L}$, 可以应用于人血清 ALP 的检测. 此 外，该探针还可应用于斑马鱼中 ALP 水平的内源性变 化监测和空间定位监测.

最近, Maitra 等 ${ }^{[45]}$ 报道了一种基于稀土 $\mathrm{Eu}(\mathrm{III})$ 金属 离子和胆酸盐水凝胶的 ALP 苂光探针 28 (Scheme 14), 其结构设计中将 1-差筇基萠的促敏剂加入到胆酸盐水凝 胶中. 在 ALP 的作用下，人工合成的底物凝胶释放自由 敏化剂，导致荧光开启，使其发出红色或洋红色的苂光.

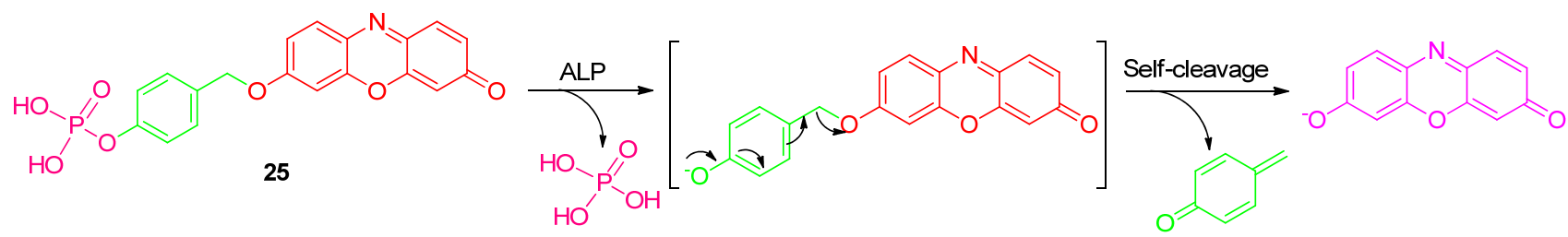

图式 12 探针 $\mathbf{2 5}$ 与 ALP 反应机理

Scheme 12 Mechanism of probe 25 reaction with ALP

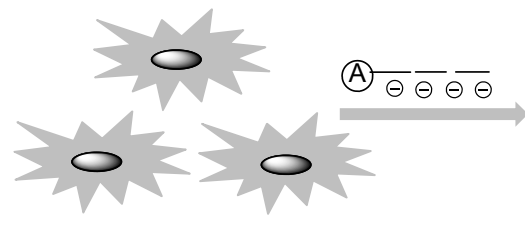

26

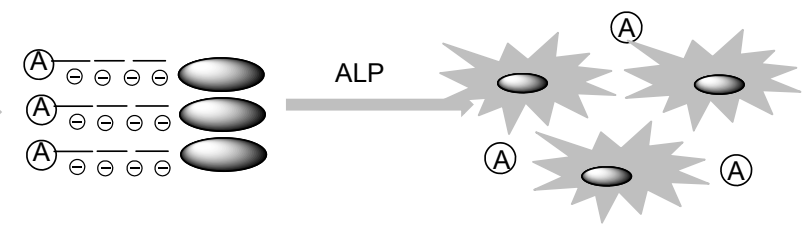

De-aggregation

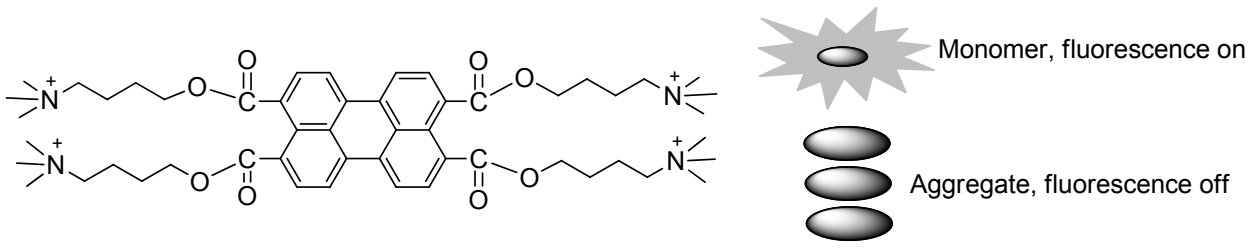<smiles>CC(C)COC1C(O)C(O)OC1n1cnc2c(N)ncnc21</smiles>

$$
\text { ATP }=(A) \bar{\ominus} \bar{\ominus} \bar{\ominus} \quad \text { Adenosine }=\text { (A) }
$$

图式 13 探针 26 与 ALP 反应机理

Scheme 13 Mechanism of probe 26 reaction with ALP 


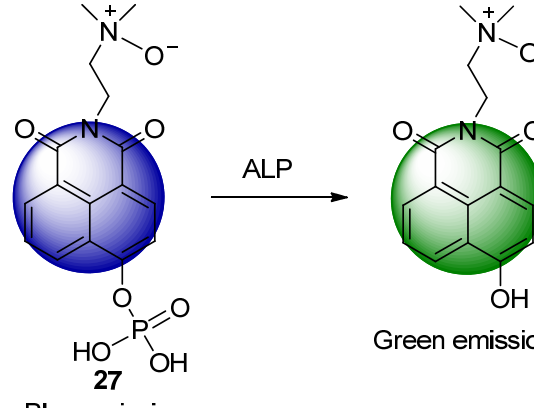

Blue emission

通过 HPLC 分析表明, 在酶催化作用下促敏剂水解导致 敏化剂的释放, 从而使胆酸盐水凝胶中的 $\mathrm{Eu}(\mathrm{III})$ 敏化. 该凝胶法的测定在纸上完成，使其具有制作简单、成本 低和使用方便等优点. 通过试验证实凝胶分析技术可用 于抑制剂篮选，该方法有望在临床上得到应用.

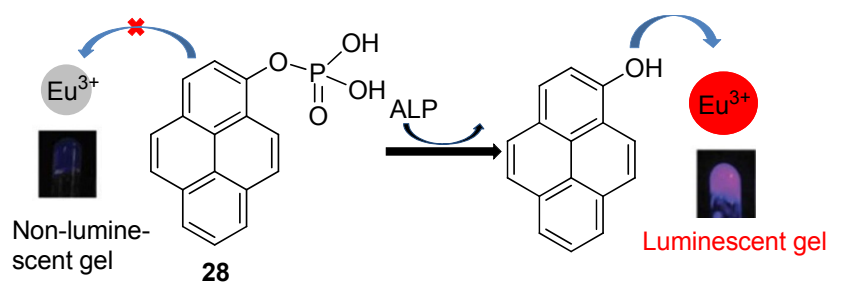

图式 14 探针 28 与 ALP 反应机理

Scheme 14 Mechanism of probe 28 reaction with ALP

\section{2 总结与展望}

经过科研工作者的不断努力, ALP 荧光探针得到了 初步发展. 主要综述了 ALP 菼光探针的设计合成、检测 以及生物应用方面的研究进展, 分别从 ESIPT/ICT、 AIE、降低给电子能力/扰乱共轭 $\pi$ 电子体系和荧光内滤 效应等发光机制方面综述. 重点介绍了 ALP 的设计合 成以及简单高效的传感检测方法. 目前, 常见的有机小 分子 ALP 荧光探针主要依据脱磷酸基团设计, 采用发 射波长不同的发光基团. 对其修饰得到不同荧光发射和 生物性能的苂光探针. 结构简单、操作方便和检测性能 优异的 ALP 荧光探针是人们所期待的. 但到目前为止, 大多数有机小分子荧光探针仍然存在合成繁琐、水溶性 差和光稳定性不佳等问题. 纳米材料因良好的生物兼容 性, 适用于开发 ALP 荧光探针, 尤其是作为药物载体在 临床上具有很大的应用前景. 在 ALP 荧光传感器的生 物应用方面, 尽管 ALP 苂光探针在细胞成像、抑制剂篎 选以及实时监测方面取得了不错的研究成果, 但现阶段 哺乳动物体内 ALP 的检测仅局限于人类血液和血清以 及小鼠体内. 在未来的一段时间里, 对于 ALP 荧光检 测, 仍然是化学和生物科研工作者研究的热点. 随着人 们对高选择性, 高灵敏性 ALP 荧光探针的不断追求, 在
不久的将来 ALP 荧光传感器必将会应用于人类疾病的 诊断及治疗.

\section{References}

[1] Coleman, J. E. Annu. Rev. 1992, 21, 411.

[2] Christenson, R. H. Clin. Biochem. 1997, 30, 573.

[3] Ooi, K.; Shiraki, K.; Morishita, Y. J. Clin. Lab. Anal. 2007, 21, 133.

[4] Wolf, P. L. J. Clin. Lab. Anal. 1994, 8, 172.

[5] Yeung, M. C.-L.; Yam, V. W.-W. Chem. Sci. 2013, 4, 2928.

[6] Liu, Y.; Schanze, K. S. Anal. Chem. 2008, 80, 8605.

[7] Ruan, C.; Wang, W.; Gu, B. Anal. Chem. 2006, 78, 3379.

[8] Iqbal, J. Anal. Biochem. 2011, 414, 226.

[9] Mwilu, S. K.; Okello, V. A.; Osonga, F. J.; Miller, S.; Sadik ,O, A. Analyst 2014, 139, 5472.

[10] Yin, C.; Huo, F.; Zhang, J. Martínez-Máñez, R.; Yang, Y.; Lv, H.; Li, S. Chem. Soc. Rev. 2013, 42, 6032.

[11] Xu, Q.; Jin, C.; Zhu, X. Chin. J. Org. Chem. 2014, 34, 647 (in Chinese). (徐勤超，金灿，朱雪慧，邢国文，有机化学, 2014, 34, 647.)

[12] Huang, X.; Zhang, F.; Zhu, L.;Choi, K. Y.; Guo, N.; Guo, J.; Tackett, K.; Anilkumar, P.; Liu, G.; Sun, Y. P.; Lee, S.; Chen, X ACS Nano 2013, 7, 5684.

[13] Wang, J.; Chu, H.; Chen, W. Chin. J. Org. Chem. 2016, 36, 2545 (in Chinese).

(王军, 初紅涛, 陈微微, 孙荣国, 有机化学, 2016, 36, 2545.)

[14] Kim, J. S.; Quang, D. T. Chem. Rev. 2007, 107, 3780.

[15] Bozdemir, O. A.; Guliyev, R.; Buyukcakir, O.; Selcuk, S.; Kolemen, S.; Gulseren. G.; Nalbantoglu, T.; Boyaci, H.; Akkaya, E. U. J. Am. Chem. Soc. 2010, 132, 8029.

[16] Hu, Q.; Zeng, F.; Yu, C.; Wu, S. Sens. Actuators, B 2015, 220, 720.

[17] Song, Z.; Kwok, R. T.; Zhao, E.; He, Z.; Hong, Y.; Lam, J, W, Y.; Liu, B.; Tang, B. Z. ACS Appl. Mater. Interfaces 2014, 6, 17245.

[18] Kim, T. I.; Kim, H.; Choi, Y.; Kim, Y. Chem. Commun. 2011, 47, 9825.

[19] Jia, Y.; Li, P.; Han, K. Chem.-Asian J 2015, 10, 2444.

[20] Fan, C.; Luo, S.; Qi, H. Luminescence 2016, 31, 423.

[21] Lu, Z.; Wu, J.; Liu, W.; Zhang, G.; Wang, P. RSC Adv. 2016, 6, 32046.

[22] Hong, Y.; Lam, J. W. Y.; Tang, B. Z. Chem. Soc. Rev. 2011, 40, 5361.

[23] Luo, J.; Xie, Z.; Lam, J. W. Y.; Cheng, L.; Chen, H.; Qiu, C.; Kwok, H. S.; Zhan, X.; Liu, Y.; Zhu, D.; Tang, B. Z. Chem. Commun. 2001, 1740.

[24] Liang, J.; Kwok, R. T.; Shi, H.; Tang, B. Z.; Liu, B. ACS Appl. Mater. Interfaces 2013, 5, 8784.

[25] Gu, X.; Zhang, G.; Wang, Z.; Liu, W.; Xiao, L.; Zhang, D. Analyst 2013, 138, 2427.

[26] Liu, H.; Lv, Z.; Ding, K. J.; Liu, X.; Yuan, L.; Chen, H.; Li, X. J. Mater. Chem. B 2013, 1, 5550.

[27] Lin, M.; Huang, J.; Zeng, F.; Wu, S. Chem.-Asian J 2018, 14, 802.

[28] Chen, Q.; Bian, N.; Cao, C.; Qiu, X. L.; Qi, A. D.; Han, B. H. Chem. Commun. 2010, 46, 4067.

[29] Zhang, W.; Yang, H.; Li, N.; Zhao, N. RSC Adv. 2018, 8, 14995.

[30] Owens, E. A.; Henary, M.; El Fakhri, G; Soo, C. H. Acc. Chem. Res. 2016, 49, 1731.

[31] Tan, Y.; Zhang, L.; Man, K. H.; Peltier, R.; Chen, G.; Zhang, H.;Zhou, L.; Wang, F.; Ho, D.; Yao, S. Q.; Hu, Y.; Sun, H. ACS Appl. Mater. Interfaces 2017, 9, 6796.

[32] Li, S. J.; Li, C. Y.; Li, Y. F.; Fei, J.; Wu, P.; Yang, B.; Yang, J. O.; Nie, S. X. Anal. Chem 2017, 89, 6854.

[33] Zhang, Q.; Li, S.; Fu, C.; Xiao, Y.; Zhang. P.; Ding, C. J. Mater. Chem. B 2019, 7, 443.

[34] Xu, L.; He, X.; Huang, Y.; Ma, P.; Jiang, Y.; Liu, X.; Tao, S.; Sun, Y.; Song, D.; Wang, X. J. Mater. Chem. B 2019, 7, 1284.

[35] Gao, Z.; Sun, J.; Gao, M.; Yu, F.; Chen, L.; Chen, Q. Sens. Actuators, $B \mathbf{2 0 1 8}, 265,565$. 
[36] Freeman, R.; Finder, T.; Gill, R.; Willner, I. Nano Lett. 2010, 10 , 2192.

[37] Li, G.; Fu, H.; Chen, X.; Gong, P.; Chen, G.; Xia, L.; Wang, H.; You, J.; Wu, Y. Anal. Chem. 2016, 88, 2720.

[38] Mao, G.; Zhang, Q.; Yang, Y.; Ji, X.; He, Z. Anal. Chim. Acta 2019, 1047, 208.

[39] Liu, H.; Li, M.; Xia, Y.; Ren, X. ACS Appl. Mater. Interfaces 2017, 9, 120.

[40] Dong, L.; Miao, Q.; Hai, Z.; Yuan, Y.; Liang, G. Anal. Chem. 2015, 87,6475 .
[41] Mei, Y.; Hu, Q.; Zhou, B.; Zhang, Y.; He, M.; Xu, T.; Li, F.; Kong, J. Talanta 2018, 176, 52.

[42] Zhang, H.; Xu, C.; Liu, J.; Li, X.; Guo, L.; Li, X. Chem. Commun. 2015, 51, 7031.

[43] Chen, J.; Jiao, H.; Li, W.; Liao, D.; Zhou, H.; Yu, C. Chem.-Asian J. 2013, 8, 8276.

[44] Hou, X.; Yu, Q.; Zeng, F.; Ye, J.; Wu, S. J. Mater. Chem. B 2015, 3, 1042.

[45] Gorai, T.; Maitra, U. J. Mater. Chem. B 2018, 6, 2143.

(Zhao, C.) 